Revue de droit comparé du travail et de la sécurité sociale

3 | 2018

La participation des travailleurs dans la grande entreprise privée et publique

\title{
Les travailleurs en marge du droit du travail
}

Pascale Lorber

\section{OpenEdition}

1 Journals

Édition électronique

URL : https://journals.openedition.org/rdctss/1935

DOI : $10.4000 /$ rdctss. 1935

ISSN : 2262-9815

Éditeur

Centre de droit comparé du travail et de la sécurité sociale

Édition imprimée

Date de publication : 1 novembre 2018

Pagination : 190-193

ISSN : $2117-4350$

\section{Référence électronique}

Pascale Lorber, « Les travailleurs en marge du droit du travail », Revue de droit comparé du travail et de la sécurité sociale [En ligne], 3 | 2018, mis en ligne le 01 novembre 2021, consulté le 13 novembre 2021. URL : http://journals.openedition.org/rdctss/1935; DOI : https://doi.org/10.4000/rdctss.1935

\section{(9) $(1) \Theta \Theta$}

Revue de droit comparé du travail et de la sécurité sociale est mise à disposition selon les termes de la Licence Creative Commons Attribution - Pas d'Utilisation Commerciale - Pas de Modification 4.0 International. 


\title{
PASCALE LORBER
}

\author{
UNIVERSITÉ DE LEICESTER
}

\section{LES TRAVAILLEURS EN MARGE DU DROIT DU TRAVAIL}

L'été a été riche en développements tant sur le plan jurisprudentiel que sur le plan politique (toujours dans le cadre du Brexit). Cette contribution se concentrera sur une décision importante de la Supreme Court (niveau supérieur de l'ordre juridictionnel) pour les travailleurs à la marge du droit du travail.

Le statut des personnes travaillant pour une entreprise est une source de contentieux permanente, et encore plus récemment, suite aux changements du monde du travail et notamment la croissance exponentielle des plateformes ${ }^{1}$. Les personnes travaillant sur demande, comme pour Uber, ou les freelancers, en dehors du secteur de la Gig Economy sont souvent dans l'ombre quand il s'agit de savoir s'ils ont accès à la protection du droit du travail en fonction de la qualification qui leur est attribuée, salariés, travailleurs ou indépendants (la matrice en droit anglais).

Les tests appliqués par les juges pour déterminer ce statut sont complexes, ouverts à interprétation, résultat de définitions statutaires imprécises du salarié ou du travailleur. Ces tests sont également influencés par le droit des contrats. L'interprétation jurisprudentielle des clauses contractuelles ne tient compte que de façon limitée de la particularité et des réalités du monde du travail ${ }^{2}$. Les conséquences pour les travailleurs concernés sont significatives puisqu'un jugement les privant du statut de salarié ou de travailleur les empêche d'accéder aux droits attachés à ces catégories juridiques (licenciement sans cause réelle et sérieuse par exemple ou droit aux congés payés).

La Supreme Court a récemment rendu des décisions qui peuvent être considérées comme des signes positifs pour ces personnes employées sous des contrats les cataloguant de travailleurs indépendants. En 2012 déjà, l'affaire Autoclenz ${ }^{3}$ avait vu des laveurs de voiture requalifiés comme travailleurs plutôt qu'indépendants suite à une argumentation qui privilégiait la réalité des relations contractuelles plutôt que le texte des clauses écrites stricto sensu. Un élément déterminant de cette réalité était la subordination. Les juges s'étaient donc montrés prêts à ignorer ce qui était considéré comme un contrat " simulé » en profession libérale (sham contract).

Une autre affaire récente, Pimlico Plumbers Ltd $v S_{\text {mith }}^{4}$, a, à nouveau, mis à jour le genre de pratiques utilisées par certains employeurs pour éviter la protection du droit du travail. Mr Smith était plombier pour la compagnie Pimlico. Pour des raisons médicales, il avait demandé une réduction du nombre de ses jours de travail, mais le refus de ce changement a aussi conduit à la fin de son contrat. La compagnie considérait Mr Smith

1 Voir cette rubrique dans cette Revue de 2017, à propos de la Taylor Review.

2 P. Leighton and M. Wynn, «Classifying employment relationships - More Sliding Doors or a Better Regulatory Framework », ILJ, 2011, n²0, p. 5.

3 Autoclenz v Belcher [2011] UKSC 41.

4 [2018] UKSC 29. 
comme un travailleur indépendant. Cette qualification a été contestée devant toutes les juridictions : de l'équivalent des prud'hommes à la Supreme Court en passant par deux Cours d'appel. Devant toutes ces instances, le contrat a été requalifié de contrat de travail.

Les juges anglais devaient se prononcer sur le statut du plombier pour savoir s'il pouvait arguer d'un licenciement sans cause réelle et sérieuse, de déductions irrégulières de son salaire, d'un manquement aux obligations concernant les congés payés et d'une discrimination sur la base d'un handicap. Le premier droit n'est accessible qu'aux salariés, alors que les autres ne nécessitent que le statut de travailleur ou d'être « en emploi » pour ce qui est de la législation sur la discrimination. Devant la Supreme Court, seule la question relative au statut de travailleur et de celui d'être " en emploi » était débattue car la notion de salarié avait été rejetée dans les décisions précédentes. Il est important de préciser que cette distinction entre salarié et travailleur est importante puisque toute la panoplie des droits n'est accessible qu'au salarié alors que le travailleur dispose d'un éventail plus réduit de protection (notamment accès au salaire minimum, à la réglementation sur les horaires de travail et les congés payés, ainsi que la protection contre la discrimination).

Le test appliqué pour le statut de travailleur consiste à démontrer deux éléments : premièrement, le travail doit être accompli personnellement par le travailleur et deuxièmement, le travailleur doit prouver que la personne pour qui il travaille n'est pas son client ou un consommateur ${ }^{5}$. La jurisprudence a principalement eu des difficultés si le contrat contient une clause de substitution qui permet au travailleur de trouver un remplaçant puisque l'élément de performance personnelle est manquant ${ }^{6}$.

Dans un premier temps, la Supreme Court a analysé la clause de substitution présente dans le contrat. Elle a noté que cette clause était conditionnée par certains paramètres imposés par Pimlico. En effet, Mr Smith ne pouvait envoyer pour une mission qu'un autre plombier « employé » comme lui, par Pimlico. Les juges ont donc considéré que la condition apportée à cette clause ne donnait pas la liberté nécessaire à Mr Smith et consistait à rendre la situation équivalente à un changement de poste ou de mission entre collègues.

Dans un deuxième temps, la Cour a dû considérer la relation entre Mr Smith et Pimlico. Elle a établi d'abord que bien que Mr Smith ait eu différentes missions successives de plomberie, il n'existait qu'un contrat « global » ou " parapluie » (umbrella contract) entre les parties. La compagnie insistait ensuite sur le fait que, pendant six ans, le plombier avait soumis une déclaration d'impôt sous le régime d'entrepreneur plutôt que celui de salarié un indice signalant une relation plus commerciale. La Supreme Court a cependant rappelé aux parties qu'un seul facteur ne peut être déterminant de la relation. L'ensemble des documents contractuels ainsi que la réalité devait être considéré. Les juges ont donc catalogué les signes caractéristiques d'une relation de travail et ceux relevant d'une relation de client ou de consommateurs. A la première se rattachaient l'obligation de porter un uniforme de la compagnie, de respecter toutes les règles administratives et financières,

5 ERA 1996 s 230(3): a worker is «an individual who has entered into or works under (or, where the employment has ceased, worked under)-(a) a contract of employment, or (b) any other contract, whether express or implied and (if it is express) whether oral or in writing, whereby the individual undertakes to do or perform personally any work or services for another party to the contract whose status is not by virtue of the contract that of a client or customer of any profession or business undertaking carried on by the individual».

6 Voir par example, Byrne Bros (Formwork) Limited v Baird [2002] IRLR 96 (EAT), Redrow Homes (Yorkshire) Ltd v Wright [2004]EWCA Civ 469, James v Redcats (Brands) Ltd [2007] ICR 1006. 
une terminologie du contrat plus apparentée à celle d'un contrat de travail, utilisant des termes de "salaires » ou licenciement. Finalement, le contrat contenait également des clauses de non-concurrence rigoureuses. Dans la seconde relation (de consommateur ou de client), les indices révélateurs incluaient l'absence de contrôle exercé par Pimlico sur le travail du plombier, le fait que le contrat autorisait Mr Smith à refuser le travail offert ou à travailler pour d'autres entreprises. Au vu de ces facteurs, la Supreme Court a décidé que la première instance avait correctement conclu qu'une relation de travail existait et que Mr Smith était un travailleur.

Cette décision a été reçue très positivement par les syndicats ${ }^{7}$. En effet, elle démontre une volonté inhabituelle, des juges d'aller au-delà du langage du contrat lorsque ces constructions contractuelles sont élaborées, semble-t-il pour échapper au droit du travail. L'insertion de clauses de substitution en particulier s'est avérée un outil utile pour manquer à la première condition nécessaire à la définition du travailleur ${ }^{8}$. Cette jurisprudence pourrait donc influencer d'autres litiges dans lesquels ces clauses ont, jusqu'à présent, été fatales à certains personnels dans le cadre de l'économie digitale. Par exemple, les cyclistes livreurs de Deliveroo se sont vus refuser l'accès à la négociation collective, seulement accessible aux travailleurs, principalement parce qu'une clause de substitution se trouvait dans leur contrat ${ }^{9}$.

Cet optimisme doit être cependant modéré. L'analyse du jugement révèle qu'une place importante est toujours consacrée à l'analyse contractuelle. Le fait que le contrat ait été rédigé de façon maladroite ou contienne des clauses imprécises (avec des références aux « salaires » ou aux licenciements) a clairement joué un rôle dans la décision finale de la Cour. II n'est pas impensable que les employeurs se tournent vers leurs avocats pour requérir une rédaction des contrats contenant une terminologie apte à satisfaire le test de relations commerciales (par exemple remplacer le terme de "salaire " par celui de «honoraire»).

Afin d'éviter que la charge d'établir le statut d'un travailleur ne pèse sur l'individu et ne soit considérée au cas par cas, les syndicats ainsi que la doctrine ${ }^{10}$ ont fait des propositions pour rendre le système plus transparent et équitable. Une simplification de la nomenclature est proposée : substituer à la triade, salarié, travailleur et entrepreneur, le binôme travailleur et entrepreneur serait préférable. La première catégorie aurait droit à l'éventail de tous les droits sans distinction, la deuxième aurait seulement certains droits. De plus, la charge de la preuve serait inversée : une présomption de statut de travailleur serait appliquée et l'entité employant devrait démontrer que le statut n'est pas celui de travailleur.

7 https://www.bbc.co.uk/news/business-44465639

8 M. Ford, Pimlico Plumbers : Cuting the Gordian Knot of Substitution Clauses ? UK Labour Law blog https://uklabourlawblog.com/2018/07/20/pimlico-plumbers-cutting-the-gordian-knot-ofsubstitution-clauses-michael-ford-qc/

9 M. Vincente, "Les coursiers Deliveroo face au droit anglais », Revue Droit du Travail, 2018, p. 515.

10 Institute of Employment Rights, A Manifesto for Labour Law, 2016. 


\section{ROYAUME-UNI}

En parallèle, le gouvernement a consulté sur le statut des personnes au travail durant le printemps $2018^{11}$. Le document indique que la distinction entre salarié, travailleur et entrepreneur sera conservée mais les définitions sont à revoir. Les propositions incluent la codification des tests jurisprudentiels et la revue des tests applicables pour le statut de salarié et de travailleur.

La direction prise par le gouvernement ne semble donc pas aller dans le sens proposé par les syndicats ou une partie de la doctrine. Une codification d'une jurisprudence complexe n'aurait pas les mêmes avantages que la simplification du régime et l'insertion d'une présomption favorable à l'individu. Dans l'attente du résultat final de ces consultations, il reste à regretter que la charge de prouver son statut continue de peser sur l'individu même si la jurisprudence est devenue plus clémente envers les travailleurs se trouvant à la marge du droit du travail.

11 Department for Business, Energy and Industrial Strategy, Employment Status Consultation (February 2018) https://assets.publishing.service.gov.uk/government/uploads/system/uploads/ attachment_data/file/679853/FINAL_-_Employment_Status_consultation_-_FOR_UPLOADING_ and_PRINTING.pdf 